\title{
Pediatric Stroke: Clinical Findings and Radiological Approach
}

\author{
Giuseppe Lanni, Alessia Catalucci, Laura Conti, Alessandra Di Sibio, \\ Amalia Paonessa, and Massimo Gallucci
}

Department of Neuroradiology, S.Salvatore Hospital, University of L'Aquila, Via Vetoio, Coppito, 67100 L'Aquila, Italy

Correspondence should be addressed to Giuseppe Lanni, giuseppe.lanni@me.com

Received 13 September 2010; Accepted 16 February 2011

Academic Editor: Halvor Naess

Copyright (C 2011 Giuseppe Lanni et al. This is an open access article distributed under the Creative Commons Attribution License, which permits unrestricted use, distribution, and reproduction in any medium, provided the original work is properly cited.

This paper focuses on radiological approach in pediatric stroke including both ischemic stroke (Arterial Ischemic Stroke and Cerebral Sinovenous Thrombosis) and hemorrhagic stroke. Etiopathology and main clinical findings are examined as well. Magnetic Resonance Imaging could be considered as the first-choice diagnostic exam, offering a complete diagnostic set of information both in the discrimination between ischemic/hemorrhagic stroke and in the identification of underlying causes. In addition, Magnetic Resonance vascular techniques supply further information about cerebral arterial and venous circulation. Computed Tomography, for its limits and radiation exposure, should be used only when Magnetic Resonance is not available and on unstable patients.

\section{Introduction}

Pediatric stroke (PS) is a relatively rare disease, having an estimated incidence of 2.5-13/100,000/year [1-4], but remains one of the most common causes of death in childhood, with a mortality rate of $0.6 / 100,000$ dead/year $[5,6]$. PS has also seriously high morbidity and long-term outcome and is nowadays gaining more interest because of its heavy consequences and costs, both personal and social. Indeed, about half of the surviving patients develop some neurologic or cognitive impairment, and just more than a quarter, epilepsy.

As in adults, PS can be ischemic or hemorrhagic; although ischemic stroke is more common, prevalence is variable $[7,8]$. Ischemic stroke includes Arterial Ischemic Stroke (AIS) and Cerebral Sinovenous Thrombosis (CSVT); Hemorrhagic Stroke (HS) includes intracerebral and subarachnoid haemorrhage [9].

AIS, both in neonatal and childhood forms, has a higher incidence among males $[5,10-14]$ and black people $[5,7]$. AIS is relapsing in 6\%-37\% of little patients [15-19] and the risk appears highest in the first 6 months after the first episode $[19,20]$. Risk factors for relapse include vascular abnormalities as first stroke cause $[19,20]$, and the presence of thrombotic risk factors [20], either isolated or as part of multiple risk factors $[20,21]$.

CSVT has an incidence of 2.6/100,000 children/year in the neonatal period and of 0.4 and $0.7 / 100,000$ children/year in childhood [22].

The incidence of hemorrhagic stroke (HS) is estimated between 0.7 to 5.1/100,000 children/year $[23,24]$ and, similar to childhood AIS, is more common in males and blacks [11, 25]. The mean age at diagnosis is 6-10 years [1].

A prompt and precise diagnosis of PS, associated to an effective management of vascular emergencies, is a crucial point to reach a correct therapy and, consequently, a positive outcome.

This paper will focus on main clinical features and radiological diagnosis of pediatric stroke.

\section{Cause and Pathophysiology}

\subsection{Ischemic Stroke}

\subsubsection{Arterial Ischemic Stroke}

AIS is defined as ischemia, infarction, or encephalomalacia in a vascular arterial distribution territory [5]. 
Classification of AIS has always been object of debate thus leading to the development of different systems. The need to subclassify patients with AIS arises from several reasons. In fact, prognosis, risk of recurrence, etiological factors, and choices of management differ between the subtypes. AIS can be divided into subgroups according to PSC (Pediatric Stroke Classification) proposed by Wraige et al. [26]. This classification is based on main etiopathological differences between pediatric and adult stroke and derives from the TOAST one, used for adult stroke [27]. PSC includes eight subtypes of AIS: (1) sickle cell disease, (2) cardioembolic, (3) Moya-Moya Syndrome, (4) cervical arterial dissection, (5) steno-occlusive cerebral arteriopathy, (6) other determined etiology, (7) multiple probable/possible etiologies, and (8) undetermined etiology.

Pediatric AIS shares with adult AIS an embolic or in situ thrombosis ground but, unlike adult stroke, degenerative vascular and chronic degenerative diseases (e.g., atherosclerosis) have very little role in its genesis. Most frequently reported risk factors for pediatric AIS are congenital or acquired heart diseases, hematologic and metabolic disorders, and vascular disorders and infections [21, 28]. Nevertheless, approximately one half of pediatric AIS occurs in children with unknown risk factors [29].

Predisposing factors for AIS are summarized in Table 1.

Vasculopathies such as Transient Cerebral Arteriopathy (TCA), arterial dissection, fibromuscular dysplasia, and Moya-Moya Disease (MMD) have been identified in 18\%$80 \%$ of children with AIS [19]. Congenital heart disease is reported in association with large vessel dissection or MMD $[30,31]$.

The most common arteriopathy associated with pediatric stroke is TCA. This is a monophasic arterial disease characterized by a unilateral focal or segmental stenosis, which involves the distal part of the internal carotid and the initial segments and branches of the anterior and/or middle cerebral artery, and is followed by complete or partial resolution [32].

The pathophysiology of TCA is uncertain but in $44 \%$ is associated with Post-Varicella Arteriopathy (PVA), an acute vasculitis caused by varicella virus infection of the arterial wall. In this cases a varicella zoster infection is identified in the 12 months prior to AIS [33, 34].

Other infectious agents, which are frequently responsible for TCA, are Parvovirus B19, CMV, Mycoplasma pneumoniae, Borrelia burgdorferi, Enterovirus, HIV, and Helicobacter pylori.

Furthermore, some patients with TCA present angiograms compatible with arterial dissections $[22,35]$.

MMD is a progressive bilateral stenosis of Willis circle arteries, which usually causes vascular insufficiency or repeated ischemic episodes, despite the development of collateral blood flow [36]. It can be primary or secondary to underlying disorders, in which case it is known as MoyaMoya Syndrome.

Primary or idiopathic MMD accounts for $12 \%$ of AIS cases [37].

The most common hematologic risk factor of stroke is Sickle Cell Disease (SCD). In this disease hemoglobin, in a deoxygenated environment, acquires higher density making red blood cells susceptible to sickle. SCD has a lot of neurologic complication, among them cerebral infarction is the most common. The stroke pathophysiology in SCD may be a combination of chronic haemolytic anemia and vasoocclusion [38]. Indeed, chronic hemolysis leads to anemia and subsequently to tissue hypoxia; anemia determines increased stress and red cell adhesion to endothelial cells with consequent injury. Damaged endothelial cells suffer alterations, including intimal hyperplasia and prothrombotic/proadhesive events. On the other hand, vaso-occlusion decreases blood flow, leading to tissue ischemia and infarction.

Alterations in vasoregulation generate a cascade precipitating acute cerebrovascular events [39].

Prothrombotic disorders such as protein $\mathrm{C}$ and protein $\mathrm{S}$ deficiency, antithrombin III deficiency, factor $\mathrm{V}$ Leiden mutation, factor XII deficiency, factor VIII deficiency, prothrombin 20210A mutations, and antiphospholipid antibodies have been found in approximately one third of children with AIS $[40,41]$.

Congenital metabolism errors such as Fabry disease, homocystinuria, organic acid disorders, ornithine transcarbamylase deficiency, and carbohydrate-deficiency glycoprotein syndrome are also well-established risk factors of children AIS [42].

Another important cause of pediatric stroke is substance abuse, especially in teenage patients. Drugs such as amphetamines, ecstasy, cocaine, and inhalants (such as glue sniffing) have been shown to be a risk for stroke from both cerebral infarct and hemorrhage. The use of marijuana has been reported as a cause of strokes with cerebellar infarction among adolescents [43]. Strokes related to these substances are thought to be caused by toxic vasculitis, transient cerebral vasoconstriction, prothrombotic effects, and exacerbation of previously unrecognized cardiovascular disease $[23,44]$. The risk of stroke has been placed as high as 6.5 per 100,000 per year in young adult drug abusers [45].

2.1.2. Cerebral Sinovenous Thrombosis. Thrombosis within the venous system results in outflow obstruction with subsequent venous congestion and persistent increase in capillary hydrostatic pressure, which drives fluid into interstitium producing edema. An increased hydrostatic pressure reduces also arterial inflow with subsequent ischemia [22].

The brain injury spectrum in CSVT varies from venous congestion to parenchymal ischemic injury, which may be cortical, subcortical, or involving deep gray matter.

Most of the parenchymal infarcts are hemorrhagic but CSVT can also lead to subarachnoid/subdural hemorrhage.

In preterm and term neonates there is also an association between CSVT and intraventricular hemorrhage (IVH) [22, 46]. Indeed, several studies demonstrate that CSVT is the most frequently identified cause of symptomatic IVH, and is associated, in term neonates, with basal ganglia or thalamic hemorrhage. 
TABLE 1: Risk factors and causes of Arterial Ischemic Stroke.

Cardiac

(1) Congenital
(a) Dysrhythmias
(b) Congenital heart disease
(c) Cardiomyopathy
(d) Cardiac tumours

(2) Acquired
(a) Cardiomyopathy
(b) Carditis
(c) Arrhythmias
(d) Artificial valves
(e) Endocarditis

(3) Iatrogenic
(a) Cardiac catheterization
(b) Cardiac surgery/cardiopulmonary bypass
(c) Carotid ligation

Hematologic

(1) Hemoglobinopathies
(a) Sickle Cell Disease
(b) Thalassemia

(2) Thrombophilia
(a) Primary
(b) Secondary

(3) Iron deficiency anemia

(4) Thrombocytopenia

Infectious

(1) Meningitis

(a) Viral, bacterial, fungal

(b) Encephalitis

Vasculitis

(1) Primary

(a) Primary angiitis of CNS

(2) Secondary

(a) Post-infectious

(i) Varicella

(ii) Other

(b) Infectious

(i) Encephalitis

(ii) Meningitis

(c) Associated with collagen vascular disease or systemic vasculitides
TABle 1: Continued.

Other vasculopathies

(1) Transient/focal cerebral arteriopathy

(2) Down syndrome

(3) Fabry disease

(4) NF1

(5) PHACE syndrome

(6) Sickle Cell Disease

(7) Moya-Moya Disease (primary)

(8) Moya-Moya Syndrome (secondary)
(a) Down syndrome
(b) NF1
(c) SCD
(d) William syndrome
(e) Post-irradiation

(9) Fibromuscular dysplasia

(10) Vasospasm
(a) Migraine
(b) Other

(11) Dissection

\section{Other}

(1) Trauma
(a) Dissection
(b) Fat/air embolism

(2) Toxins/Drugs
(a) Cocaine
(b) L-asparaginase
(c) Oral contraceptives

(3) Metabolic
(a) Shock/dehydration
(b) Carbohydrate deficient glycoprotein syndrome
(c) Homocysteinuria

Deep venous thrombosis can be accompanied by haemorrhage into the ventricles, as a result of blockage, and hypertension in the deep venous drainage system [47].

Presumed perinatal ischemic stroke is a subgroup of perinatal stroke and encompasses imaging-confirmed focal infarction, which may be venous or arterial, presenting after the neonatal period [48].

Perinatal Venous Infarction (PVI) is one of these periventricular infarction syndromes and is an underrecognized cause of congenital hemiplegia $[29,35]$.

Risk factors and causes of CSVT are summarized in Table 2.

2.2. Hemorrhagic Stroke. Most nontraumatic hemorrhagic strokes are intracerebral hemorrhages, which may also 
TABLE 2: Risk factors and causes of CSVT.

\section{(1) General}
(a) Dehydration
(b) Infection
(c) Fever
(d) Hypoxic-ischemic injury
(e) Post lumbar puncture

(2) Head and neck infections
(a) Otitis media and mastoiditis
(b) Meningitis
(c) Sinusitis
(d) Upper respiratory tract infection

(3) Other head and neck disorders
(a) Head injury
(b) Post intracranial surgery
(c) Hydrocephalus ( \pm ventriculoperitoneal shunt)

(4) Anemia
(a) Iron deficiency
(b) Sickle cell disease
(c) Thalassemia
(d) Autoimmune hemolytic anemia
(e) Paroxysmal nocturnal hemoglobinuria

(5) Autoimmune disorders
(a) Behçet disease
(b) Systemic lupus erythematosus
(c) Antiphospholipid antibody syndrome
(d) Inflammatory bowel disease (ulcerative colitis, Crohn disease)
(e) Thyrotoxicosis
(f) Cushing syndrome
(g) Idiopathic thrombocytopenic purpura

(6) Malignancy
(a) Leukemia
(b) Lymphoma
(c) Central nervous system tumors

(7) Cardiac disease
(a) Cyanotic congenital heart disease
(b) Post-operative
(c) Postcatheterization

(8) Renal disease
(a) Nephrotic syndrome
(b) Hemolytic-uremic syndrome

(9) Drugs
(a) L-Asparaginase
(b) Oral contraceptives
(c) Corticosteroids
(d) Epoetin- $\alpha$

(10) Chromosomal disorders

(a) Down syndrome

(11) Metabolic conditions

(a) Diabetic ketoacidosis

(b) Homocystinuria
TABLE 3: Risk factors and causes of Hemorrhagic Stroke.

Genetic vasculopathy
(1) Arteriovascular malformation
(2) Intracranial aneurysm
(3) Cavernous angioma
(4) Neurocutaneous disorders
(5) Ehlers-Danlos syndrome
(6) Moya-Moya Syndrome
(7) Fibromuscular dysplasia
(8) Fabry disease

Hematologic disorders

(1) Hemoglobinopathy

(2) Platelet disorders

(3) Coagulopathy

(4) Hypofibrinogenemia

Trauma

Hypertension

(1) Congenital adrenal hyperplasia

(2) Stimulant drug use

(3) Coarctation of aorta

originate in or extend into the intraventricular, subdural, or subarachnoid space $[43,44]$.

Risk factors and causes of HS are summarized in Table 3.

Studies about children with HS show that first cause is vascular malformations, which are responsible of 5\%-29\% of cerebral hemorrhages, whereas other causes are hematological disorders, such as thrombocytopenia or hemophilia, and neoplasms $[43,44]$.

The most common vascular malformations are arteriovenous malformations (AVMs), aneurysms, and cavernous malformations [43, 44]. Aneurysms and hypertension, although commonly associated with adult HS, are an infrequent cause in children [45].

Most AVMs are diagnosed in patients between 20 and 40 years, but about $18 \%$ to $20 \%$ will become symptomatic during childhood $[44,49]$. In a series of 37 children with AVMs, $70 \%$ presented with HS; the annual risk of HS in children with an AVM is 3.2\%, and the risk of recurrent relapsing is $6 \%-33 \%$ in the first year after the initial bleed $[50,51]$.

The incidence of intracranial aneurysms in children is about 1 per million per year, substantially less than the adult rate $[43,44]$. About $1 \%$ to $2 \%$ of aneurysms will become symptomatic in childhood, mainly with HS.

\section{Clinical Presentation}

The clinical presentation of AIS is extremely various, depending on age, cause, and involved vascular territory $[9,23]$. Usually, embolic stroke tends to present suddenly, whereas thrombosis may have a more gradual onset $[9,23]$. Focal neurologic deficits (cranial nerve palsies, hemiparesis, and hemisensory loss) are the most common presentation 
of AIS in children. Seizures, headache, language and speech difficulties, and altered mental status are also possible [9, 52]. Stroke in the posterior circulation can present as ataxia, vertigo, and vomiting. In infancy, typical presentation includes seizure, lethargy, and/or apnea often without focal neurologic deficits [9]. Pediatric stroke can also impact behavioural and psychiatric functions.

The clinical presentation of HS depends on the child's age and the size and location of the hemorrhage. Main signs and symptoms include headache, vomiting, seizures, impaired consciousness, and/or focal neurologic deficits [44].

The clinical manifestations of CSVT are nonspecific, may be subtle and may overlap with predisposing conditions such as infection and dehydration. Seizures, altered levels of consciousness and encephalopathy, focal neurologic deficits and diffuse neurologic symptoms (headache, nausea, and vomiting) may result; presentation with pseudotumor cerebri and isolated headache has also been documented [22].

\section{Radiological Diagnosis}

Key points in the diagnosis of childhood stroke are causal investigation, laboratory tests, and imaging studies.

Radiological exam is often the first step in the evaluation of an acutely ill child.

The two imaging modalities to be used in emergency are Computed Tomography (CT) and/or Magnetic Resonance (MR).

Which is the first-choice imaging modality is still object of debate.

Our experience, derived from acute and followup observation of 41 patients, permits to consider MR imaging (MRI) as first-line emergency examination [4].

In fact MRI, especially with the integration of diffusionweighted (DWI) and perfusion-weighted (PWI) imaging, is optimal for diagnosing stroke. DWI is the most sensitive tool in the diagnosis of cytotoxic edema, thus offering the unique possibility of diagnosing an acute ischemic stroke also in cases with apparently normal CT and MRI conventional sequences [4] (Figure 1). Moreover, perfusion imaging can offer a prognostic value: in acute stroke, in fact, it allows to determinate the volume of tissue at risk and the vascular distribution of the ischemia; the level of perfusion to the ischemic tissues may also help to determine the relative benefits and risks of a given therapy [53]. It is well known, in fact, that the perfusion/diffusion "mismatch" reflects the difference between the ischemic core and surrounding penumbra: the areas of DWI abnormalities with decreased $\mathrm{ADC}$ values are assumed to have suffered irreversible injury, by the time of patient examination; hence, an area of lowered PWI and normal DWI is an index of the penumbra, or tissue that has potentially reversible ischemia. These values had been recently considered as useful screening for systemic or intra-arterial thrombolysis approach in adulthood.

The role and the indications of intra-arterial thrombolysis in pediatric stroke has not been definitively assessed yet because a very few cases have been reported and clinical trials are in course. Anyway, as reported in literature, outcomes of children with stroke who underwent thrombolysis, suggest that this treatment may also be beneficial in pediatric population. Controlled randomized trials are needed in order to determine the appropriate dosage, safety, and efficacy of intra-arterial thrombolysis; feasibility of mechanical thrombolysis should be evaluated as well [54-57].

On the other hand, the use of MR angiography (MRA) allows the detection and location of intracerebral arterial lesions in a noninvasive way, although the characterization of the type of lesion is the main limit of MRA (circumferential clefts, intimal flaps, intraluminal thrombi, or tapering of middle cerebral artery branches) [58].

MR spectroscopy (MRS) and DWI with MRA could also, in selected cases, increase the sensitivity of MRI in the detection of ischemia and infarction [59]. According to English guidelines, cross-sectional brain imaging is mandatory in children presenting with clinical stroke, and brain MRI is recommended for investigation in these patients [25]. Brain MRI should be undertaken as soon as possible after presentation. If brain MRI will not be available within 48 hours, CT is an acceptable initial alternative [25]. Brain imaging should be undertaken urgently in children with clinical stroke who have a depressed level of consciousness at presentation or whose clinical status is deteriorating, in which case CT scanning should be used.

If the identified infarct has unusual features, more specific venous imaging investigations may then be applied by the radiologist as necessary. In the first instance, noninvasive options such as MR venography (MRV) or CT venography (CTV) are preferred over intra-arterial digital subtraction angiography (IADSA) [25].

MRI and MRV are actually the preferred methods for investigation of CSVT because of their sensitivity and specificity and for the excellent anatomical correlation between venous drainage system and location of parenchymal infarcts. DWI and PWI may play a role in detecting venous congestion in cerebral venous thrombosis and in the differentiation of cytotoxic and vasogenic edema, but do not differentiate venous from arterial infarction.

The diagnosis is established by demonstrating a lack of flow in the cerebral veins with or without typical images of brain infarction [22].

MRI and MRV allow to demonstrate both the infarct and the clot within the vessels. On MRI, the thrombus is easily recognizable in the subacute phase, when it appears hyperintense on T1-weighted images. In the acute phase, the thrombus is isointense with brain on T1-weighted images and hypointense on T2-weighted images. This appearance can be mistaken for flowing blood (Figure 2), but MRV will demonstrate an absence of flow in the thrombosed sinus. T2*-weighted images seems to be more sensitive than T1- or T2-weighted or Fluid Attenuated Inversion Recovery (FLAIR) images in demonstrating venous thrombosis and associated hemorrhage [60].

When MRI exam cannot be performed, unenhanced CT may detect deep venous thrombosis as linear densities in the expected locations of the deep and cortical veins. As the thrombus becomes less dense, contrast may demonstrate the "empty delta" sign, a filling defect, in the posterior part of 


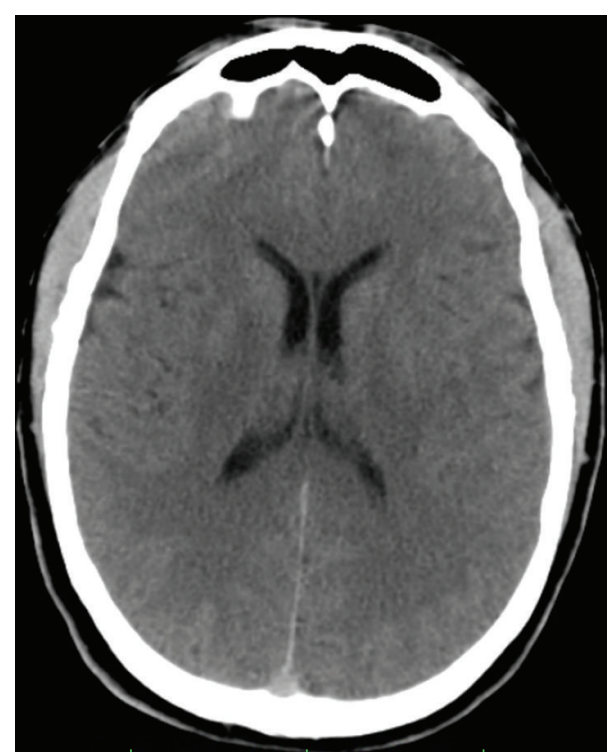

(a)

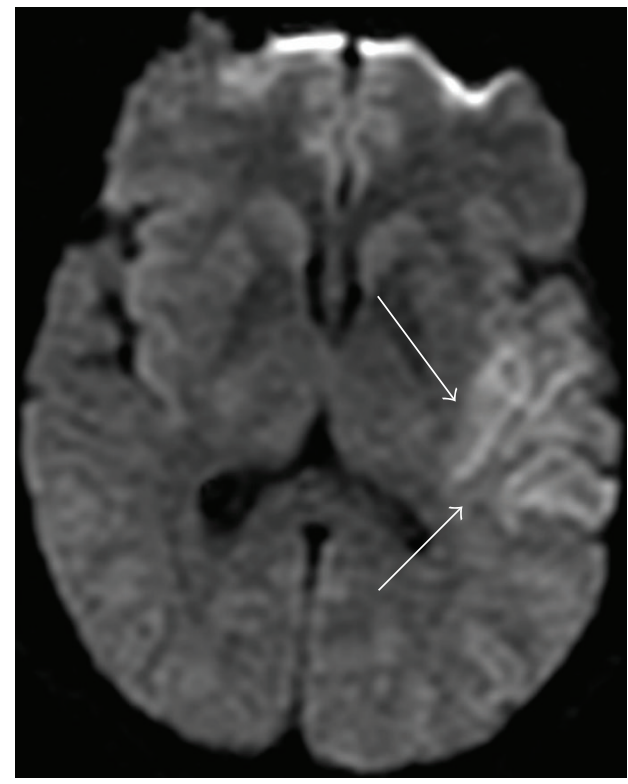

(c)

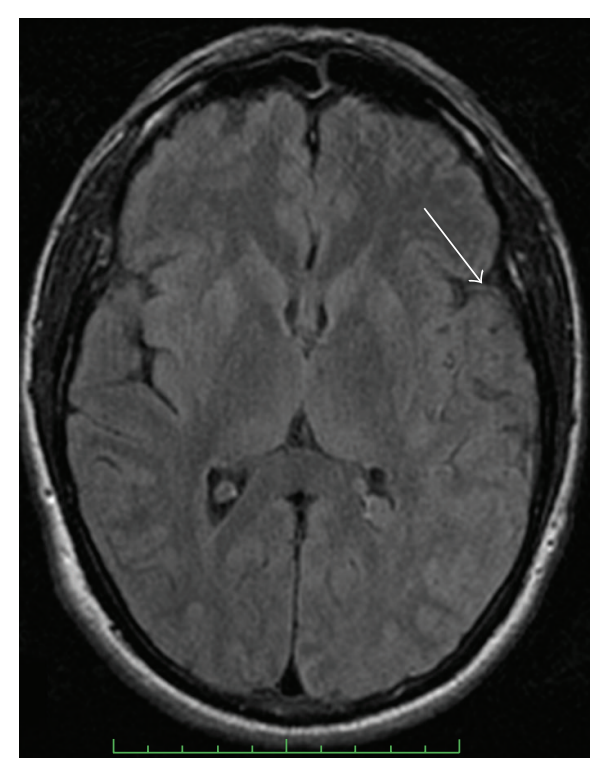

(b)

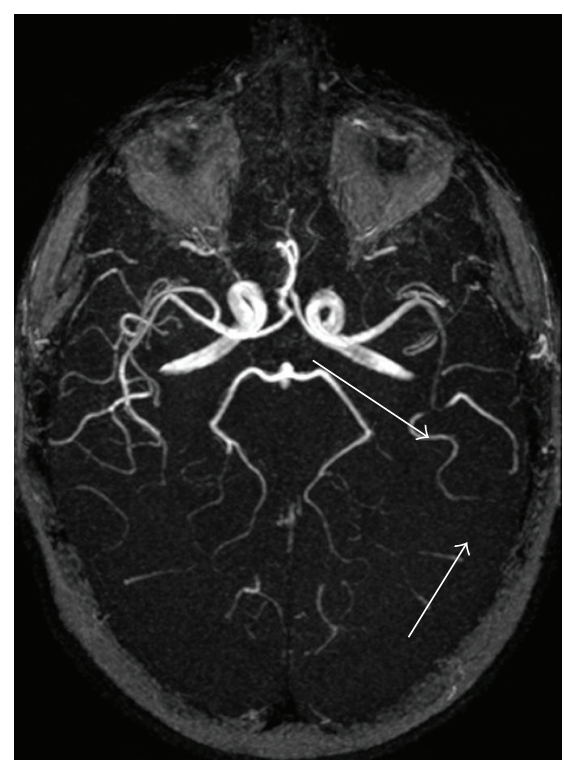

(d)

FIGURE 1: A 14-year-old male with right-sided hemiparesis started 3 hours before MRI. (a) Plain CT scan does not show significant density abnormality. (b) MRI T2-weighted Fluid Attenuated Inversion Recovery image does not show significant signal intensity abnormality of cerebral parenchyma even if hyperintensities of distal branches of middle cerebral artery are visible and suggest vessel occlusion (arrow). (c) MRI Diffusion-weighted image shows bright signal of left insular and temporal cortex indicating cytotoxic edema (arrows). (d) 3D-Time of Flight MR-angiogram shows poor representation of distal branches of left middle cerebral artery (arrows).

the sagittal sinus (Figure 2). However, CT scan with contrast misses the diagnosis of CSVT in up to $40 \%$ of patients [22] so CTV can be a reasonable in-depth examination.

In every case, imaging of the cervical and proximal intracranial arterial vasculature should be performed in all children with AIS and imaging of the cervical vasculature to exclude arterial dissection should be undertaken within 48 hours of presentation with AIS [25]. Transthoracic cardiac echocardiography should be undertaken within 48 hours after presentation in all children with AIS [25].
The limit usually attributed to MRI, related to the length of the examination, can be easily overcome by the use of dedicated "fast protocols".

Another limit classically attributed to MRI, is its presumed reduced sensitivity to acute bleeding, but different studies $[4,61]$ report that $\mathrm{MR}$ is always able to identify the acute bleeding and to distinguish spontaneous intracranial hematoma from the hemorrhagic transformation of ischemic stroke. Moreover, even when intracranial bleeding is diagnosed with $\mathrm{CT}$, identification of the cause of 


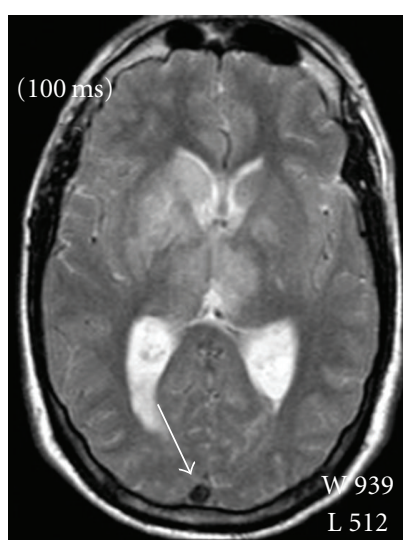

(a)

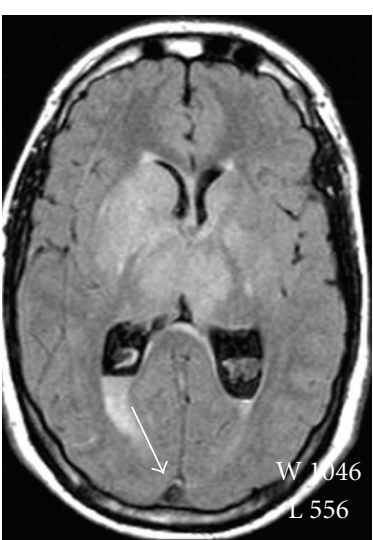

(b)

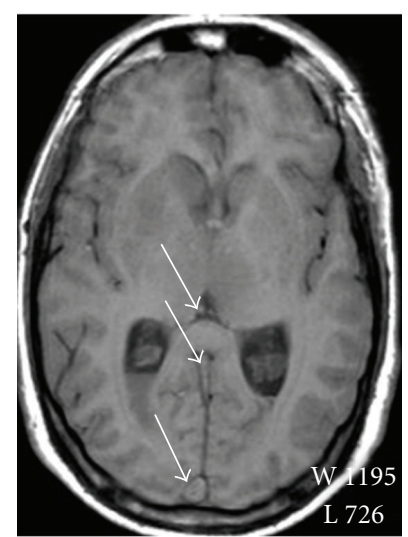

(c)

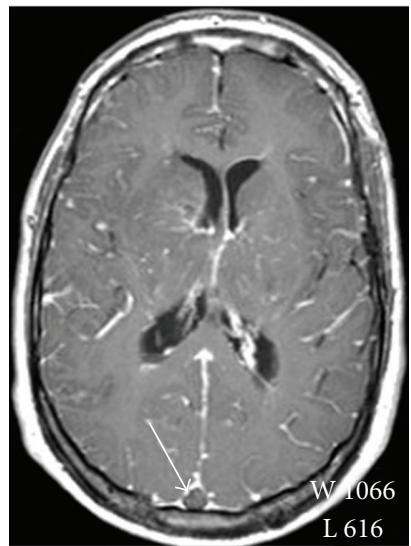

(d)

FIGURE 2: A 16-year-old female with progressively worsening headache, generalized seizures on the 4th day and coma on the 5th day. MRI exam was performed on the 5th day. MRI T2-weighted Turbo Spin Echo (a) and T2-weighted Fluid Attenuated Inversion Recovery images (b) show altered signal intensity involving bilaterally medial thalamus, lentiform nucleus, and caudate nucleus. Intraventricular bleeding is present and hemorrhagic infarct involving right frontoparietal junction (not shown) was also detected. Superior sagittal sinus and internal cerebral veins seem to have regular "flow void" signal (arrows), due to the dark signal of subacute thrombus. MRI T1-weighted images before (c) and after (d) administration of contrast agent better show occlusion of superior sagittal sinus, vein of Galen and internal cerebral veins (arrows). Visible advanced signs of venous stasis and the so-called "delta sign" (arrow) (d).

hemorrhage is often difficult without MR. In fact, MR is rapidly able to recognize the cause of the hemorrhage, and identify the presence of vascular malformations eligible to surgical or interventional treatment (Figures 3 and 4) [4].

The "real" limitation of MRI is probably the need for cooperative patients or for sedation. In most cases, anaesthesiological support, for both sedation and clinical monitoring, is required; CT, otherwise, is much faster and can be more easily performed even on unstable patients.

Considering that many pediatric patients are comatose or already under general sedation before being transferred to diagnostic imaging, MRI can be proposed as the firstchoice examination even in the acute phase of a stroke [4]. Moreover, in selected cases where differential diagnosis includes nonischemic pathology, use of paramagnetic contrast agent can be used safely. CT should be performed only on uncooperative/unstable patients and when MRI is not available; after overcoming of the critical phase it is anyway recommended that MRI is performed in the immediate followup [4].

Moreover, even if CT is very sensitive and specific in the detection of hemorrhagic lesions, in the acute phase of cerebral infarction, parenchymal abnormalities may be subtle on CT, and early/small lesion(s) in the posterior fossa can be missed [9].

CT angiography (CTA) is a noninvasive method for evaluation of intra, and extracranial circulation. CTA performed in early stages of cerebral ischemia may provide crucial information regarding cerebral circulation [62]. Disadvantages of CTA include radiation exposure, use of intravenous contrast, and the difficulty in timing the contrast bolus in small children [9].

As mentioned before also MRI and MRA supply precise information regarding intra, and extracranial vascular lesions, especially in craniocervical arterial dissections. The presence on intramural hematoma can be assessed with cross-sectional T1-, T2-, and PD-weighted images while a global visualization of vessel structure can be obtained with MRA. Time-of-flight (TOF) MRA can demonstrate a T1 hyperintense intramural clot, whereas phase-contrast (PC) MRA and contrast-enhanced (CE) MRA demonstrate only the vessel lumen [63-65]. Diagnosis of cervical artery dissection with cross-sectional images depends on the characteristics of the intramural hematoma, surrounding structures and MR sequences. An optimal exam should include T1- and T2weighted with and without fat suppression and PD-weighted sequences. Subacute hematoma appears on fat suppressed T1-weighted images as a crescent-shaped hyperintense area around an eccentric flow void corresponding to the vessel lumen. Acute dissection can be missed on fat suppressed T1-weighted images because isointense hematoma may be obscured when surrounded by isointense tissues [64, 65].

The gold standard for the definitive assessment of cerebral vasculature is IADSA which should be considered in children when pathology of small distal artery is suspected and with an unexplained infarct or hemorrhage not elucidated by MRI or MRA evaluation [66].

IADSA is used to diagnose MMD, showing bilateral stenosis of the internal carotid artery and the development of a collateral network (rete mirabile) with the appearance of the typical pattern known as "puff of smoke" (Figure 5), or in suspected dissection $[9,36]$. MRI and MRA, and in some cases IADSA, should be repeated in cases of identified arteriopathies 3 to 6 months after the initial investigation, and again at 6 to 12 months in most patients, and at the appearance of any new clinical manifestations to evaluate for additional subclinical infarcts and progression or regression of previously identified vasculopathy $[9,67]$. 


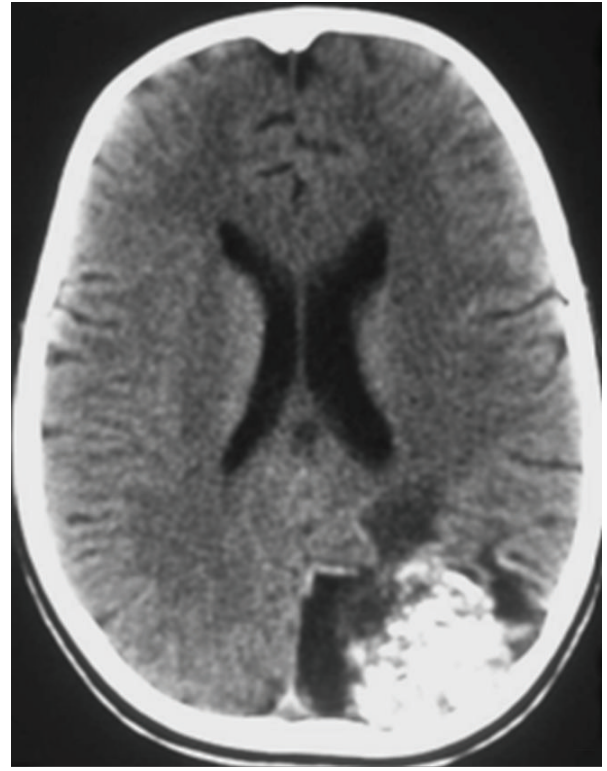

(a)

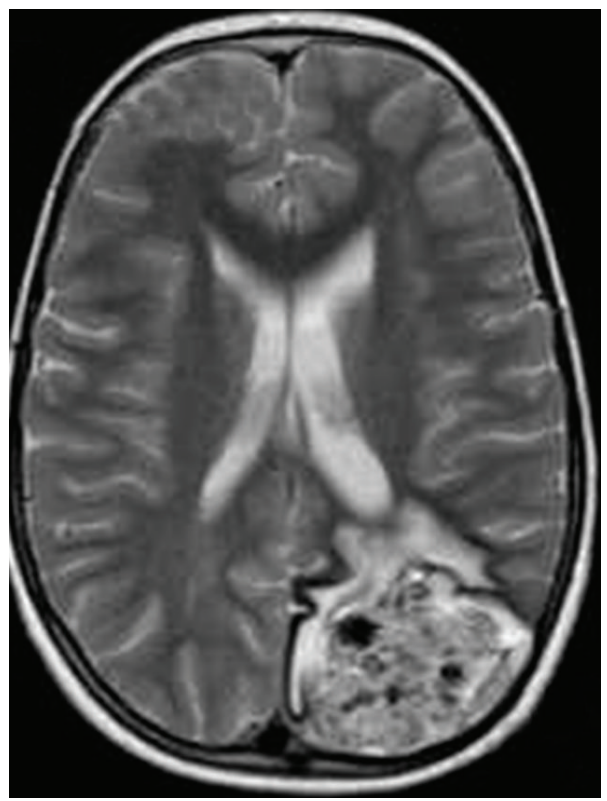

(c)

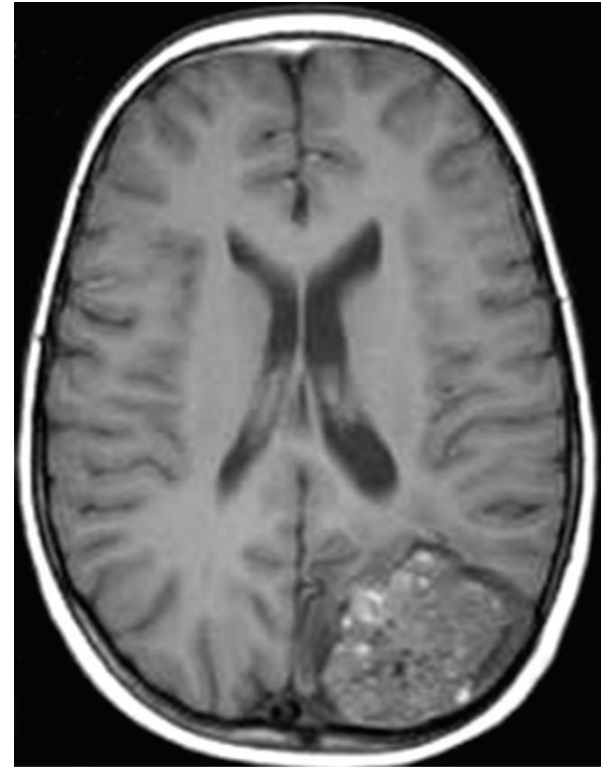

(b)

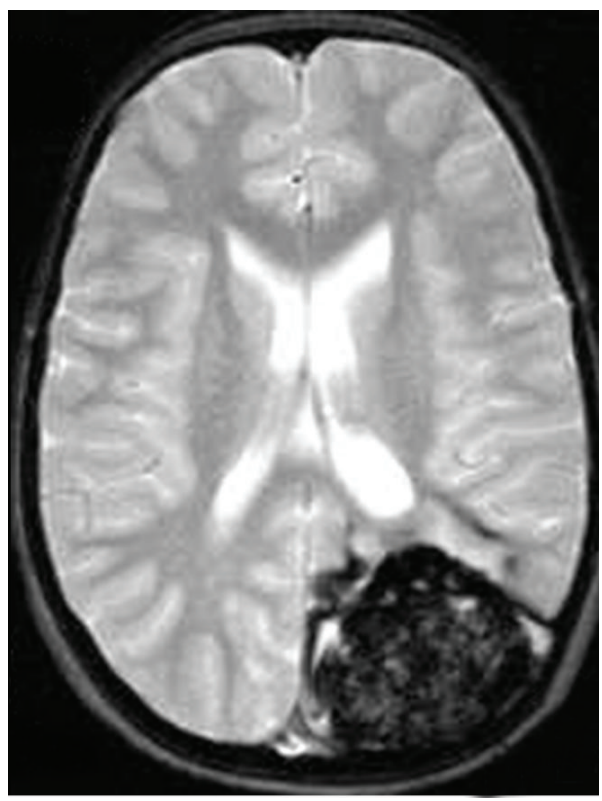

(d)

FIGURE 3: A 5-year-old male with sudden onset of visual defect followed by generalized seizures without previous hepileptic history. (a) Plain CT scan showing grossly calcified extracerebral occipital mass with loss of brain tissue. (b) MRI T1-weighted image shows typical "salt and pepper" mixed signal in the mass. (c) MRI T2-weighted Turbo Spin Echo image shows the same mass with features indicating hemosiderine rim and different stages of blood clot. (d) MRI T2*-weighted Fast Field Echo image confirms susceptibility sensitive signal consistent with blood degradation products and different stages of bleeding. All MRI features suggested the diagnosis of giant, mostly extracerebral cavernoma, surgically confirmed.

\section{Conclusions}

Pediatric stroke is a dramatic disease that requires urgent multidisciplinary competence and approach. In both cases of ischemic and hemorrhagic origin, the radiological approach to be obtained in emergency leads to the first screening and the first therapeutic possibility. Our and others experience suggest that in cooperating children as well as in comatose one or in patients already under general anesthesia, MRI should be considered as the first diagnostic step, offering a complete diagnostic set of information. In cases in which MRI is not available or in noncooperative children, it is recommended to perform a CT scan as first diagnostic step, followed as soon as possible by an MRI study. 


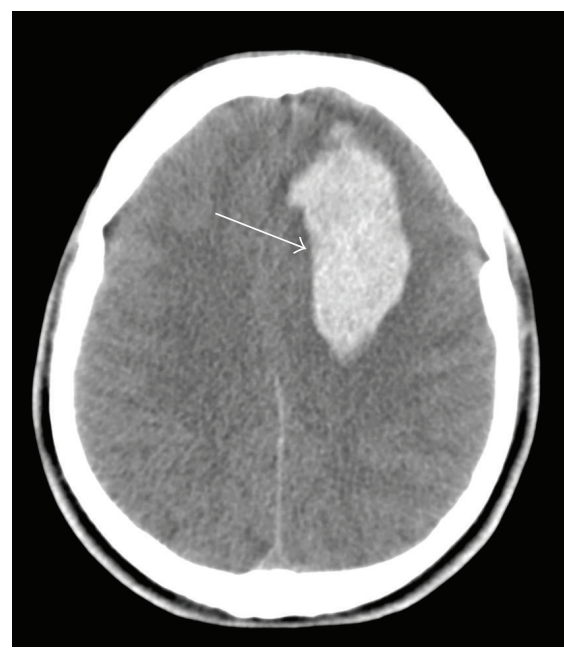

(a)

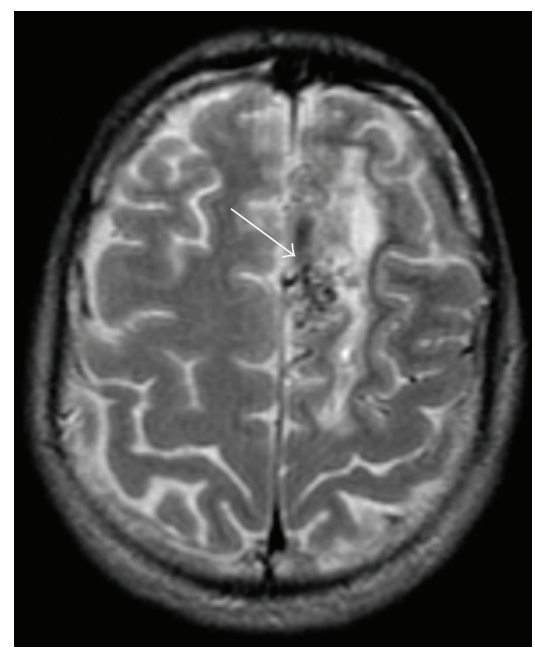

(b)

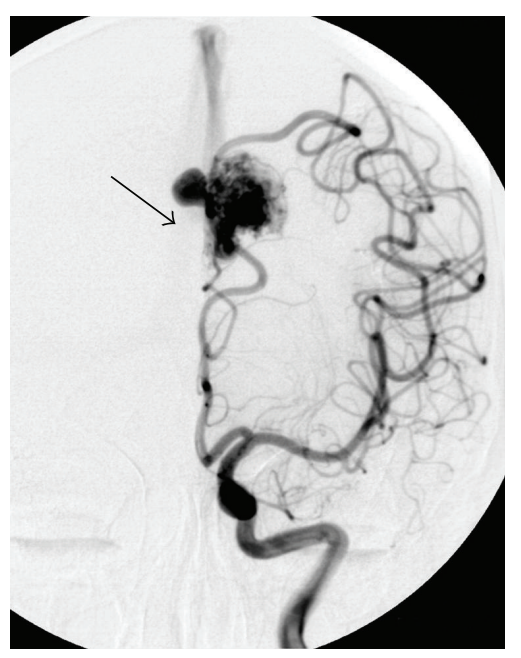

(c)

FIgURe 4: A 17-year-old male with headache, neck stiffness, and right hemiparesis. (a) Plain CT scan shows left frontal intraparenchymal hemorrhage (arrow). (b) MRI T2-weighted Turbo Spin Echo performed 4 days after surgical evacuation of hemorrhagic lesion shows the cause of bleeding: an arteriovenous malformation (arrow). (c) Intra-arterial digital subtraction angiography confirms and better evaluates the arteriovenous malformation (arrow).

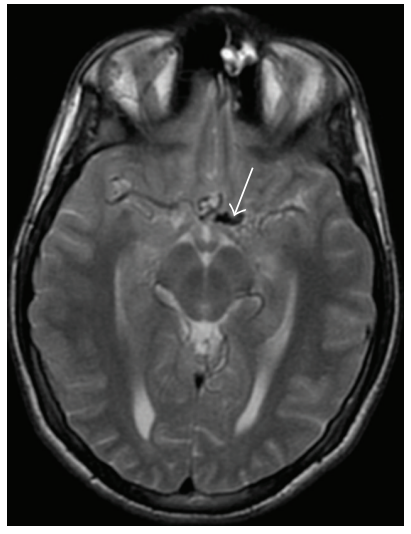

(a)

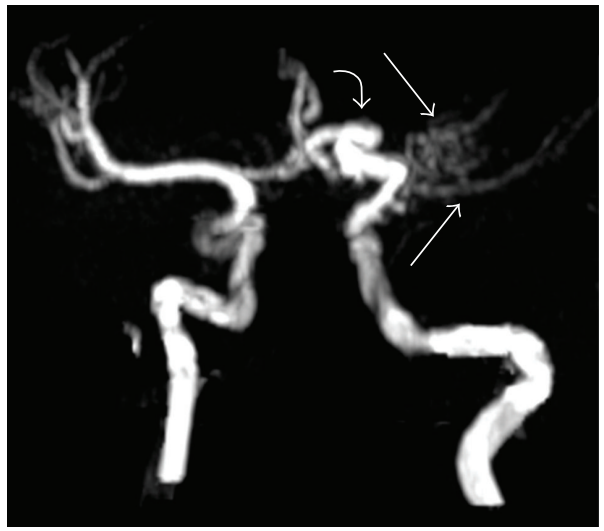

(b)

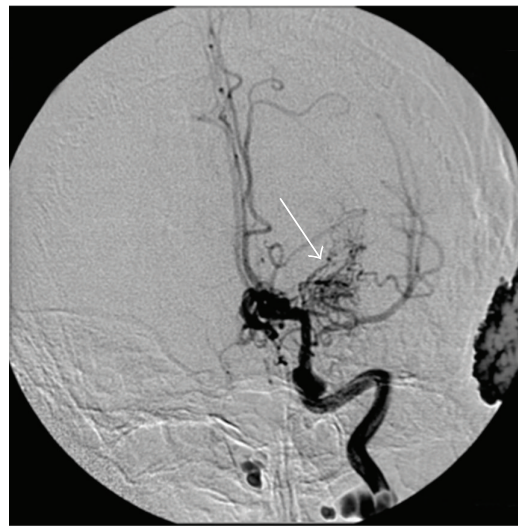

(c)

FIgURE 5: A 15-year-old male presenting with transient right hemiparesis. (a) MRI T2-weighted Turbo Spin Echo image shows vascular abnormality in left portion of the circle of Willis (arrow). (b) MR-angiogram shows a progressive reduction of diameter of the supraclinoid internal carotid artery associated with occlusion of the origin of left middle cerebral artery (curved white arrow) and presence of tiny newly formed compensatory network (white arrows). (c) Intra-arterial digital subtraction angiography better shows the occlusion of left middle cerebral artery and the typical presence of tortuous tiny vessels appearing as the so-called "puff of smoke" ("moya-moya") (arrow), formed to compensate for the supply blockage.

\section{References}

[1] M. Giroud, M. Lemesle, J. B. Gouyon, J. L. Nivelon, C. Milan, and R. Dumas, "Cerebrovascular disease in children under 16 years of age in the city of Dijon, France: a study of incidence and clinical features from 1985 to 1993," Journal of Clinical Epidemiology, vol. 48, no. 11, pp. 1343-1348, 1995.

[2] B. S. Schoenberg, J. F. Mellinger, and D. G. Schoenberg, "Cerebrovascular disease in infants and children: a study of incidence, clinical features, and survival," Neurology, vol. 28, no. 8 , pp. $763-768,1978$.
[3] J. Broderick, G. T. Talbot, E. Prenger, A. Leach, and T. Brott, "Stroke in children within a major metropolitan area: the surprising importance of intracerebral hemorrhage," Journal of Child Neurology, vol. 8, no. 3, pp. 250-255, 1993.

[4] A. Paonessa, N. Limbucci, E. Tozzi, A. Splendiani, and M. Gallucci, "Radiological strategy in acute stroke in children," European Journal of Radiology, vol. 74, no. 1, pp. 77-85, 2010.

[5] N. Friedman, "Pediatric stroke: past, present and future," Advances in Pediatrics, vol. 56, no. 1, pp. 271-299, 2009. 
[6] E. Arias, R. N. Anderson, H. C. Kung, S. L. Murphy, and K. D. Kochanek, "Deaths: final data for 2001," National Vital Statistics Reports, vol. 52, no. 3, pp. 1-115, 2003.

[7] H. J. Fullerton, Y. W. Wu, S. Zhao, and S. C. Johnston, "Risk of stroke in children: ethnic and gender disparities," Neurology, vol. 61, no. 2, pp. 189-194, 2003.

[8] D. B. Zahuranec, D. L. Brown, L. D. Lisabeth, and L. B. Morgenstern, "Is it time for a large, collaborative study of pediatric stroke?" Stroke, vol. 36, no. 9, pp. 1825-1829, 2005.

[9] M. Lopez-Vicente, S. Ortega-Gutierrez, C. Amlie-Lefond, and M. T. Torbey, "Diagnosis and management of pediatric arterial ischemic stroke," Journal of Stroke and Cerebrovascular Diseases, vol. 19, no. 3, pp. 175-183, 2010.

[10] M. R. Golomb, H. J. Fullerton, U. Nowak-Gottl, and G. DeVeber, "Male predominance in childhood ischemic stroke: findings from the international pediatric stroke study," Stroke, vol. 40, no. 1, pp. 52-57, 2009.

[11] V. Ganesan, M. Prengler, M. A. McShane, A. M. Wade, and F. J. Kirkham, "Investigation of risk factors in children with arterial ischemic stroke," Annals of Neurology, vol. 53, no. 2, pp. 167173, 2003.

[12] C. Barnes, F. Newall, J. Furmedge, M. Mackay, and P. Monagle, "Arterial ischaemic stroke in children," Journal of Paediatrics and Child Health, vol. 40, no. 7, pp. 384-387, 2004.

[13] M. Steinlin, I. Pfister, J. Pavlovic et al., "The first three years of the Swiss neuropaediatric stroke registry (SNPSR): a population-based study of incidence, symptoms and risk factors," Neuropediatrics, vol. 36, no. 2, pp. 90-97, 2005.

[14] G. A. DeVeber, D. MacGregor, R. Curtis, and S. Mayank, "Neurologic outcome in survivors of childhood arterial ischemic stroke and sinovenous thrombosis," Journal of Child Neurology, vol. 15, no. 5, pp. 316-324, 2000.

[15] E. L. L. M. De Schryver, L. J. Kappelle, A. JennekensSchinkel, and A. C. B. Peters, "Prognosis of ischemic stroke in childhood: a long-term follow-up study," Developmental Medicine and Child Neurology, vol. 42, no. 5, pp. 313-318, 2000.

[16] B. Chung and V. Wong, "Pediatric stroke among Hong Kong Chinese subjects," Pediatrics, vol. 114, no. 2, pp. e206-212, 2004.

[17] B. J. P. Delsing, C. E. Catsman-Berrevoets, and I. M. Appel, "Early prognostic indicators of outcome in ischemic childhood stroke," Pediatric Neurology, vol. 24, no. 4, pp. 283-289, 2001.

[18] H. J. Fullerton, Y. W. Wu, S. Sidney, and S. C. Johnston, "Risk of recurrent childhood arterial ischemic stroke in a population-based cohort: the importance of cerebrovascular imaging," Pediatrics, vol. 119, no. 3, pp. 495-501, 2007.

[19] R. Sträter, S. Becker, A. von Eckardstein et al., "Prospective assessment of risk factors for recurrent stroke during childhood-a 5-year follow-up study," The Lancet, vol. 360, no. 9345 , pp. 1540-1545, 2002.

[20] S. Lanthier, L. Carmant, M. David, A. Larbrisseau, and G. De Veber, "Stroke in children: the coexistence of multiple risk factors predicts poor outcome," Neurology, vol. 54, no. 2, pp. 371-378, 2000.

[21] H. J. Fullerton, J. S. Elkins, and S. C. Johnston, "Pediatric stroke belt: geographic variation in stroke mortality in US children," Stroke, vol. 35, no. 7, pp. 1570-1573, 2004.

[22] N. Dlamini, L. Billinghurst, and F. J. Kirkham, "Cerebral venous sinus (sinovenous) thrombosis in children," Neurosurgery Clinics of North America, vol. 21, no. 3, pp. 511-527, 2010.
[23] J. K. Lynch and C. J. Han, "Pediatric stroke: what do we know and what do we need to know?" Seminars in Neurology, vol. 25, no. 4, pp. 410-423, 2005.

[24] C. J. Earley, S. J. Kittner, B. R. Feeser et al., "Stroke in children and sickle-cell disease: Baltimore-Washington cooperative young stroke study," Neurology, vol. 51, no. 1, pp. 169-176, 1998.

[25] Paediatric Stroke Working Group, "Stroke in childhood: clinical guidelines for diagnosis, management and rehabilitation," Royal College of Physicians, November 2004, http://bookshop .rcplondon.ac.uk/contents/f98c6540-a541-4bed-837d-ef293ac458bf.pdf.

[26] E. Wraige, K. R. E. Pohl, and V. Ganesan, "A proposed classification for subtypes of arterial ischaemic stroke in children," Developmental Medicine and Child Neurology, vol. 47, no. 4, pp. 252-256, 2005.

[27] H. P. Adams Jr., B. H. Bendixen, L. J. Kappelle et al., "Classification of subtype of acute ischemic stroke: definitions for use in a multicenter clinical trial. TOAST. Trial of Org 10172 in Acute Stroke Treatment," Stroke, vol. 24, no. 1, pp. 35-41, 1993.

[28] U. Nowak-Göttl, G. Günther, K. Kurnik, R. Sträter, and F. Kirkham, "Arterial ischemic stroke in neonates, infants, and children: an overview of underlying conditions, imaging methods, and treatment modalities," Seminars in Thrombosis and Hemostasis, vol. 29, no. 4, pp. 405-414, 2003.

[29] T. M. Carlin and A. Chanmugam, "Stroke in children," Emergency Medicine Clinics of North America, vol. 20, no. 3, pp. 671-685, 2002.

[30] F. J. Kirkham and A. M. Hogan, "Risk factors for arterial ischemic stroke in childhood," CNS Spectrums, vol. 9, no. 6, pp. 451-464, 2004.

[31] F. Kirkham, G. Sébire, M. Steinlin, and R. Sträter, "Arterial ischaemic stroke in children: review of the literature and strategies for future stroke studies," Thrombosis and Haemostasis, vol. 92, no. 4, pp. 697-706, 2004.

[32] G. Sébire, "Transient cerebral arteriopathy in childhood," The Lancet, vol. 368, no. 9529, pp. 8-10, 2006.

[33] G. Losurdo, R. Giacchino, E. Castagnola et al., "Cerebrovascular disease and varicella in children," Brain and Development, vol. 28 , no. 6 , pp. 366-370, 2006.

[34] G. Sébire, L. Meyer, and S. Chabrier, "Varicella as a risk factor for cerebral infarction in childhood: a case-control study," Annals of Neurology, vol. 45, no. 5, pp. 679-680, 1999.

[35] K. P. J. Braun, M. M. M. Bulder, S. Chabrier et al., "The course and outcome of unilateral intracranial arteriopathy in 79 children with ischaemic stroke," Brain, vol. 132, no. 2, pp. 544-557, 2009.

[36] J. A. Gosalakkal, "Moyamoya disease: a review," Neurology India, vol. 50, no. 1, pp. 6-10, 2002.

[37] Y. Mineharu, K. Takenaka, H. Yamakawa et al., "Inheritance pattern of familial moyamoya disease: autosomal dominant mode and genomic imprinting," Journal of Neurology, Neurosurgery and Psychiatry, vol. 77, no. 9, pp. 1025-1029, 2006.

[38] R. J. Adams, K. Ohene-Frempong, and W. Wang, "Sickle cell and the brain," Hematology. American Society of Hematology Education Program, pp. 31-46, 2001.

[39] C. A. Hillery and J. A. Panepinto, "Pathophysiology of stroke in sickle cell disease," Microcirculation, vol. 11, no. 2, pp. 195208, 2004.

[40] V. Ganesan, M. A. McShane, R. Liesner, J. Cookson, I. Hann, and F. J. Kirkham, "Inherited prothrombotic states and ischaemic stroke in childhood," Journal of Neurology Neurosurgery and Psychiatry, vol. 65, no. 4, pp. 508-511, 1998. 
[41] G. DeVeber, P. Monagle, A. Chan et al., "Prothrombotic disorders in infants and children with cerebral thromboembolism," Archives of Neurology, vol. 55, no. 12, pp. 1539-1543, 1998.

[42] J. F. Meschia, T. G. Brott, and R. D. Brown, "Genetics of cerebrovascular disorders," Mayo Clinic Proceedings, vol. 80, no. 1, pp. 122-132, 2005.

[43] L. V. Gabis, R. Yangala, and N. J. Lenn, “Time lag to diagnosis of stroke in children," Pediatrics, vol. 110, no. 5, pp. 924-928, 2002.

[44] S. Goodman and S. Pavlakis, "Pediatric and newborn stroke," Current Treatment Options in Neurology, vol. 10, no. 6, pp. 431-439, 2008.

[45] J. K. Lynch, S. Pavlakis, and G. DeVeber, "Treatment and prevention of cerebrovascular disorders in children," Current Treatment Options in Neurology, vol. 7, no. 6, pp. 469-480, 2005.

[46] G. Sébire, B. Tabarki, D. E. Saunders et al., "Cerebral venous sinus thrombosis in children: risk factors, presentation, diagnosis and outcome," Brain, vol. 128, no. 3, pp. 477-489, 2005.

[47] L. A. Ramenghi, B. J. Gill, S. F. Tanner, D. Martinez, R. Arthur, and M. I. Levene, "Cerebral venous thrombosis, intraventricular haemorrhage and white matter lesions in a preterm newborn with factor V (Leiden) mutation," Neuropediatrics, vol. 33, no. 2, pp. 97-99, 2002.

[48] T. F. Barron, D. A. Gusnard, R. A. Zimmerman, and R. R. Clancy, "Cerebral venous thrombosis in neonates and children," Pediatric Neurology, vol. 8, no. 2, pp. 112-116, 1992.

[49] C. Di Rocco, G. Tamburrini, and M. Rollo, "Cerebral arteriovenous malformations in children," Acta Neurochirurgica, vol. 142, no. 2, pp. 145-158, 2000.

[50] E. R. Smith, W. E. Butler, and C. S. Ogilvy, "Surgical approaches to vascular anomalies of the child's brain," Current Opinion in Neurology, vol. 15, no. 2, pp. 165-171, 2002.

[51] S. Chabrier, B. Husson, P. Lasjaunias, P. Landrieu, and M. Tardieu, "Stroke in childhood: outcome and recurrence risk by mechanism in 59 patients," Journal of Child Neurology, vol. 15, no. 5, pp. 290-294, 2000.

[52] J. A. Zimmer, B. P. Garg, L. S. Williams, and M. R. Golomb, "Age-related variation in presenting signs of childhood arterial ischemic stroke," Pediatric Neurology, vol. 37, no. 3, pp. 171$175,2007$.

[53] R. E. Latchaw, H. Yonas, G. J. Hunter et al., "Guidelines and recommendations for perfusion imaging in cerebral ischemia: a scientific statement for healthcare professionals by the writing group on perfusion imaging, from the Council on Cardiovascular Radiology of the American Heart Association," Stroke, vol. 34, no. 4, pp. 1084-1104, 2003.

[54] G. Tsivgoulis, J. A. Horton, J. M. Ness et al., "Intravenous thrombolysis followed by intra-arterial thrombolysis and mechanical thrombectomy for the treatment of pediatric ischemic stroke," Journal of the Neurological Sciences, vol. 275, no. 1-2, pp. 151-153, 2008.

[55] C. Amlie-Lefond, A. K. C. Chan, A. Kirton et al., "Thrombolysis in acute childhood stroke: design and challenges of the thrombolysis in pediatric stroke clinical trial," Neuroepidemiology, vol. 32, no. 4, pp. 279-286, 2009.

[56] M. Arnold, M. Steinlin, A. Baumann et al., "Thrombolysis in childhood stroke report of 2 cases and review of the literature," Stroke, vol. 40, no. 3, pp. 801-807, 2009.

[57] I. Q. Grunwald, S. Walter, M. G. Shamdeen et al., "New mechanical recanalization devices-the future in pediatric stroke treatment," Journal of Invasive Cardiology, vol. 22, no. 2, pp. 63-66, 2010.
[58] B. Husson, G. Rodesch, P. Lasjaunias, M. Tardieu, and G. Sébire, "Magnetic resonance angiography in childhood arterial brain infarcts: a comparative study with contrast angiography," Stroke, vol. 33, no. 5, pp. 1280-1285, 2002.

[59] A. Venkataraman, P. B. Kingsley, P. Kalina et al., "Newborn brain infarction: clinical aspects and magnetic resonance imaging," CNS Spectrums, vol. 9, no. 6, pp. 436-444, 2004.

[60] M. Selim, J. Fink, I. Linfante, S. Kumar, G. Schlaug, and L. R. Caplan, "Diagnosis of cerebral venous thrombosis with echoplanar T2*-weighted magnetic resonance imaging," Archives of Neurology, vol. 59, no. 6, pp. 1021-1026, 2002.

[61] A. C. Y. Liu, N. Segaren, T. S. C. Cox et al., "Is there a role for magnetic resonance imaging in the evaluation of non-traumatic intraparenchymal haemorrhage in children?" Pediatric Radiology, vol. 36, no. 9, pp. 940-946, 2006.

[62] D. S. Atkinson Jr., "Computed tomography of pediatric stroke," Seminars in Ultrasound, CT and MRI, vol. 27, no. 3, pp. 207-218, 2006.

[63] C. Oppenheim, O. Naggara, E. Touzé et al., "High-resolution $\mathrm{mr}$ imaging of the cervical arterial wall: what the radiologist needs to know," Radiographics, vol. 29, no. 5, pp. 1413-1431, 2009.

[64] M. H. Rodallec, V. Marteau, S. Gerber, L. Desmottes, and M. Zins, "Craniocervical arterial dissection: spectrum of imaging findings and differential diagnosis," Radiographics, vol. 28, no. 6, pp. 1711-1728, 2008.

[65] C. M. Flis, H. R. Jäger, and P. S. Sidhu, "Carotid and vertebral artery dissections: clinical aspects, imaging features and endovascular treatment," European Radiology, vol. 17, no. 3, pp. 820-834, 2007.

[66] V. Ganesan, L. Savvy, W. K. Chong, and F. J. Kirkham, "Conventional cerebral angiography in children with ischemic stroke," Pediatric Neurology, vol. 20, no. 1, pp. 38-42, 1999.

[67] G. Sébire, H. Fullerton, E. Riou, and G. DeVeber, "Toward the definition of cerebral arteriopathies of childhood," Current Opinion in Pediatrics, vol. 16, no. 6, pp. 617-622, 2004. 


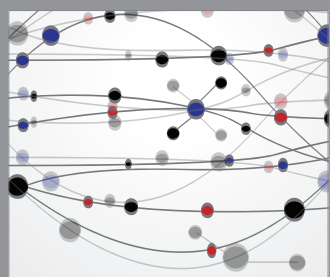

The Scientific World Journal
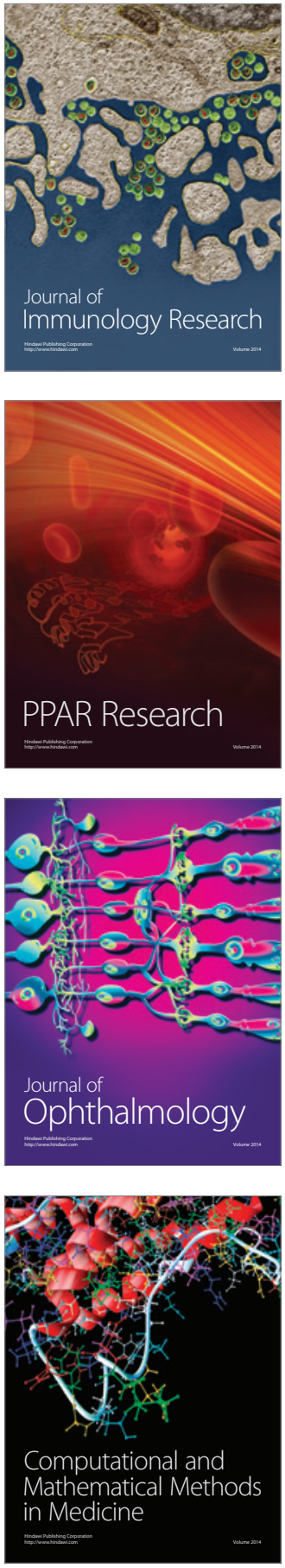

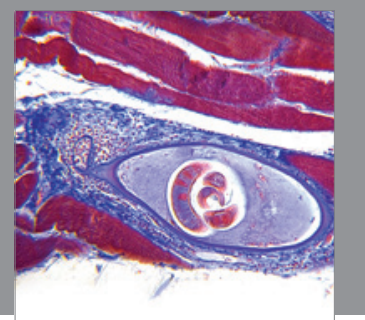

Gastroenterology

Research and Practice
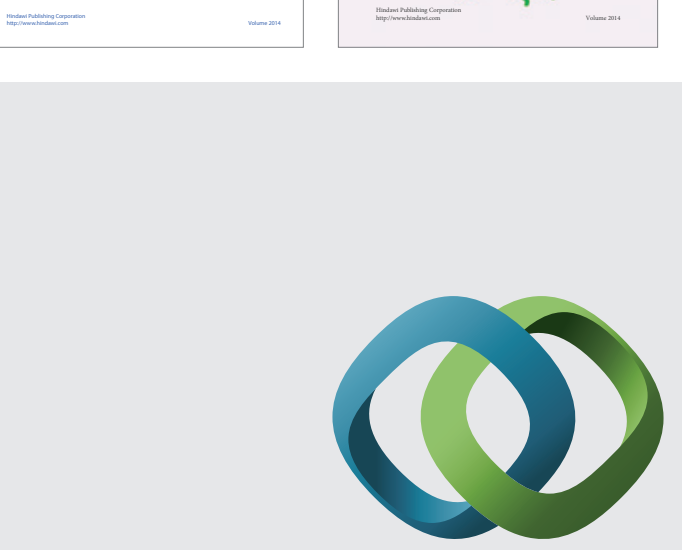

\section{Hindawi}

Submit your manuscripts at

http://www.hindawi.com
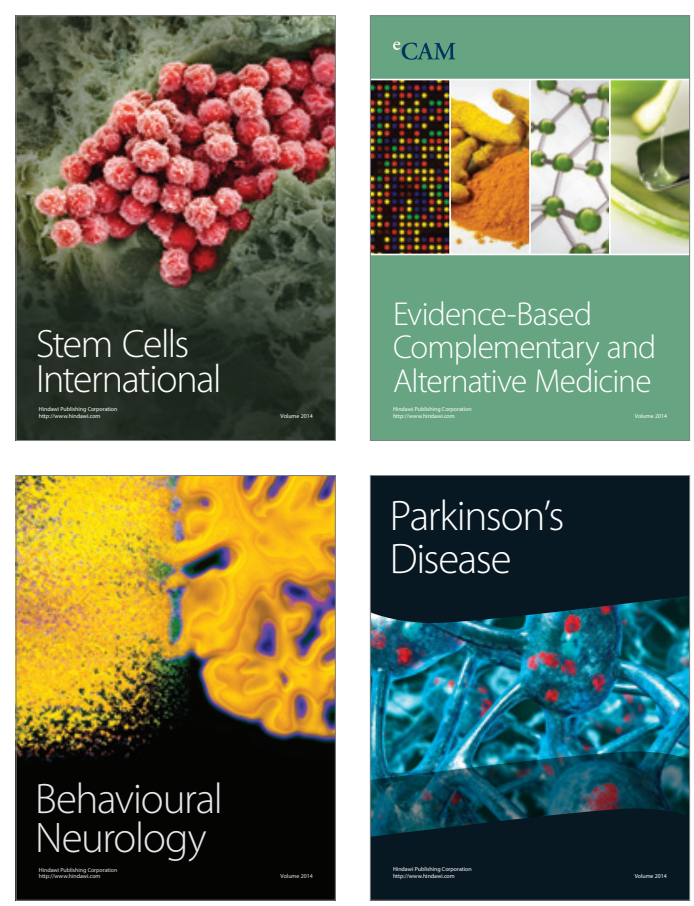

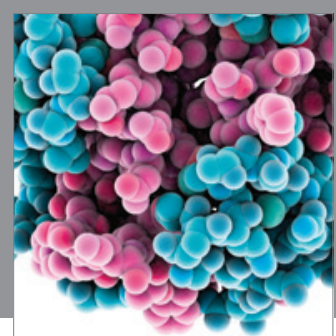

Journal of
Diabetes Research

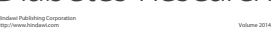

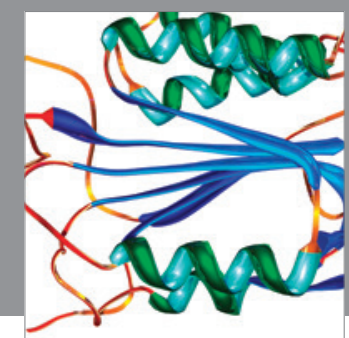

Disease Markers
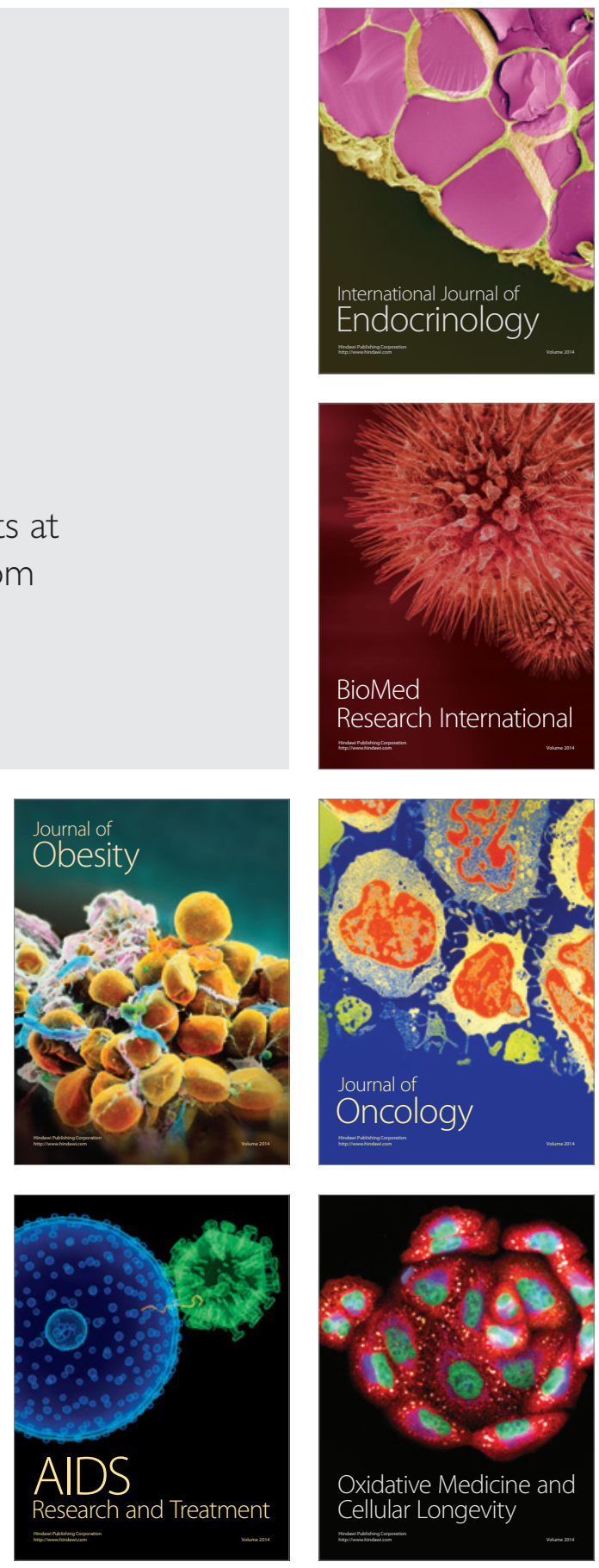